\title{
Análisis de la sobrecarga del cuidador del paciente en diálisis peritoneal
}

\author{
Cristina Elena Carmona Moriel, Cristina Nolasco Monterroso, Lucía Navas Santos, José Caballero Romero, \\ Purificación Morales Medina
}

Universidad de Córdoba, Unidad de Gestión Clínica de Nefrología, Hospital Universitario Reina Sofía. Córdoba. España

\section{Resumen}

Introducción: Un hecho diferencial de la diálisis peritoneal ambulatoria es la necesidad de colaboración de un cuidador principal que se responsabiliza del tratamiento del paciente. Esto unido al carácter crónico de esta patología y posible dependencia del paciente provoca cambios en el estilo de vida del cuidador principal siendo por tanto, una persona expuesta a la sobrecarga.

Objetivo: Identificar el grado de sobrecarga de los cuidadores de pacientes en diálisis peritoneal ambulatoria en el Hospital Reina Sofía de Córdoba y su relación con la calidad de vida y la dependencia del paciente.

Pacientes y métodos: Se realizó un estudio observacional descriptivo y transversal. Se estudiaron 18 pacientes en tratamiento con diálisis peritoneal ambulatoria y sus respectivos cuidadores. Se incluyó a todos los cuidadores y pacientes que aceptaron colaborar, previo consentimiento informado. Se utilizó el cuestionario SF-36 para evaluar la calidad de vida del paciente junto con el test de Zarit y un cuestionario de sobrecarga de los cuidadores de pacientes en diálisis peritoneal ambulatoria. Con éste último, también de identificó el nivel de dependencia del enfermo desde el criterio del cuidador.

Resultados: Respecto a la calidad de vida, se encuentra una disminución leve de algunas de las dimensiones de la población general para la misma edad. No se encontró sobrecarga en la mayoría de los cuidadores principales con ninguno de los instrumentos usados. Se encontró una correlación directa entre ambos instrumentos. En cuanto al nivel de dependencia, un $83 \%$ de pacientes eran independientes y un $17 \%$ de pacientes eran dependientes.

Correspondencia:

Cristina Elena Carmona Moriel

C/Abejar n 36 casa 2. 14002 Córdoba

E-mail:n12camoc@uco.es
Conclusión: A la vista de estos resultados, podemos concluir que, al menos en la muestra estudiada se encuentra una baja proporción de cuidadores con sobrecarga. De igual forma, los pacientes presentan un nivel bajo de dependencia con una CVRS similar a la población general de referencia excepto en la función física.

PALABRAS CLAVE

- DIÁLISIS PERITONEAL

- SOBRECARGA DEL CUIDADOR

- DEPENDENCIA

- CALIDAD DE VIDA

- CUESTIONARIO DE SOBRECARGA DEL CUIDADOR

Analysis of caregiver burden in peritoneal dialysis patients

Abstract

Introduction: A differential fact ambulatory peritoneal dialysis is the need for collaboration of a primary caregiver who is responsible for the patient's treatment. This coupled with the chronic nature of this disease and possible dependence of the patient causes changes in the lifestyle of the primary caregiver being therefore a person exposed to overload.

Objective: To identify the degree of overload of carers of patients in ambulatory peritoneal dialysis at the Reina Sofia Hospital in Cordoba and its relationship to quality of life and dependence of the patient.

Patients and Methods: A descriptive and crosssectional study. We studied 18 patients treated with continuous ambulatory peritoneal dialysis and their caregivers. We included all caregivers and patients who agreed to participate, prior informed consent. 
The SF-36 questionnaire was used to evaluate the quality of life of patients with test Zarit and questionnaire overload caregivers of patients in ambulatory peritoneal dialysis. With the latter, also it identified the level of dependence of the patient from the judgment of the caregiver.

Results: Regarding the quality of life, there is a slight decrease in some of the dimensions of the general population for the same age. No overload found in most primary caregivers with any of the instruments used. A direct correlation was found between the two instruments. Regarding the level of dependence, $83 \%$ of patients were independent and $17 \%$ of patients were dependent.

Conclusion: In view of these results, we can conclude that, at least in the sample is a low proportion of caregivers overload. Likewise, patients have a low level of dependence similar to the general population reference HRQOL except in physical function.

\section{KEYWORDS}

- PERITONEAL DIALYSIS

- CAREGIVER BURDEN

- DEPENDENCY

- QUALITY OF LIFE

- CAREGIVER BURDEN QUESTIONNAIRE

\section{Introducción}

En los últimos años, el incremento de la esperanza de vida española ha aumentado el número de pacientes con enfermedades crónicas como la diabetes, la ateroesclerosis o la hipertensión arterial ${ }^{1}$. Esto ha dado lugar a que aumente la incidencia y prevalencia de la enfermedad renal crónica (ERC) que en su estadío final, necesitará tratamiento renal sustitutivo (TRS)2.

En nuestro país, según el Informe de Diálisis y Trasplantes del 2013 de la Sociedad Española de Nefrología, la incidencia global es de 145,3 por millón de personas (pmp). Si lo dividimos por grupos de edades, existe una gran diferencia entre las personas menores de 45 años y el resto de grupos de edad. La incidencia de las personas menores de 45 años es del $12 \%$. Mientras que en los grupos de personas mayores de 45-64 es de un 32\%, los de $65-74$ es de un $26 \%$ y en los mayores de 75 es del $30 \%$. Observando el resultado de estos datos, la incidencia de IRCT tiene un importante aumento a partir de los 45 años. En relación con la prevalencia, la incidencia global es de 1416,4 pmp. También existe una considerable diferencia en los grupos de edades, en la cual, hay un $15 \%$ de menores de 45 años con respecto al $39 \%$ mayores de 45 , el $23 \%$ mayores de 64 y el $22 \%$ mayores de 75 años. Por lo tanto, también hay un aumento considerable de pacientes con IRCT a partir de los 45 años. En cuanto a la distribución de los pacientes por TRS, un $43 \%$ de pacientes se encuentran en tratamiento con hemodiálisis (HD), un $6 \%$ se encuentra en tratamiento de la diálisis peritoneal (DP) y el $51 \%$ se encuentran con un trasplante renal funcionante ${ }^{3}$. Estos datos son muy similares a los del registro europeo. Según la Asociación Renal Europea - Diálisis y Trasplantes en Europa (ERA - EDTA), España se encuentra en el vigésimo lugar de incidencia de pacientes con IRCT. No obstante, respecto a la prevalencia se encuentra en el sexto lugar ${ }^{4}$.

Dentro de las TSR, se encuentra la DP que ofrece al paciente una gran autonomía. Sin embargo en ocasiones se indica en aquellas personas, que precisamente por su falta de autonomía y/fragilidad, no es candidato a otro tipo de terapia como la HD o el trasplante. Esto da a lugar a que un porcentaje importante con DP sean personas dependientes que precisen la ayuda de un cuidador para cubrir total o parcialmente sus necesidades y para realizar la técnica y los cuidados específicos que corresponden a ésta ${ }^{5}$.

Es de resaltar que este procedimiento tiene como principal característica que el paciente asume su propio tratamiento en el domicilio, asistido en la mayoría de los casos por un familiar previamente entrenado. Este entrenamiento se produce en el servicio hospitalario y es realizado por enfermería, por lo cual son pacientes muy dependientes de su enfermera referente. Suele durar aproximadamente una semana y hasta que paciente y/o familiar no han adquirido una serie de conocimientos y habilidades no inician la técnica en su domicilio6.

Cuando los pacientes tienen ciertas limitaciones físicas o psíquicas (ancianos, diabéticos con problemas de visión, etc.) es el familiar el que asume el tratamiento incluidos los cuidados, lo cual no es complejo, pero puede sobrecargar psíquicamente a éste, al hacerse responsables del mismo. Por este motivo es por el que el familiar del paciente en DP puede ser susceptible de sufrir sobrecarga ${ }^{7}$. 
El concepto de carga se remonta a los años sesenta, cuando Grady Sainsbury en 1963 se interesaron en conocer cómo la convivencia con los pacientes psiquiátricos afectaba a la familia y a la vida doméstica ${ }^{8}$. Esta "carga del cuidador" se definió como "el conjunto de problemas de orden físico, psíquico, emocional, social o económico que pueden experimentar los cuidadores de adultos incapacitados $1 / 9$.

En esta misma línea, Zarit y otros colaboradores con $\neg$ sideraron la "carga de los cuidadores" como la clave para estudiar el desgaste ocasio $\neg$ nado por el cuidado de pacientes. Las investigaciones relaciona $\neg$ das con dicha carga han dado origen a diversos instrumentos de medición que han tenido en cuenta otras dimensiones como la carga objeti $\neg v a$ y subjetiva ${ }^{10}$. La objetiva que mide tanto el grado de dedicación del familiar al proporcionar los cuidados como el impacto que produce en su vida al proporcionarlos (cambio en las vacaciones, salir menos, etcétera); la sobrecarga subjetiva se refiere al impacto psíquico asociado a los cuidados que puede provocar agotamiento y otras dificultades para manejar la situación de cuidados (estrés, nerviosismo, ansiedad, etcétera) ${ }^{11}$.

Si observamos algunos estudios sobre la sobrecarga del familiar en TRS, encontramos un estudio del 2011, realizado en América del Norte, en el cual el $57 \%$ de los pacientes en HD tenían cuidadores no renumerados. Se asoció la carga del cuidador con una peor Calidad de Vida Relacionada con la Salud (CVRS) y depresión, según la percepción del paciente. El $50 \%$ de los pacientes sentían que sus cuidadores se sentían sobrecargados al proporcionar los cuidados ${ }^{12}$. Otro estudio más reciente, realizado en nuestro medio, en pacientes en HD, encontró una sobrecarga intensa del $33 \%$ en los cuidadores de pacientes con algún grado de dependencia, siendo los cuidadores de los pacientes menos dependientes los que presentaron menos sobrecarga en el cuidador ${ }^{13}$.

Respecto a la salud mental del cuidador, podemos destacar que en un estudio realizado en Cádiz en 2013 , el $45 \%$ de los cuidadores de HD sentían una sobrecarga leve o moderada y el 52\% tenían síntomas somáticos y ansiedad e insomnio. El 76\% presentaba síntomas depresivos ${ }^{14}$.

Los familiares que conviven con un paciente con DP, tienen cambios en su estilo de vida, día a día. Éstos están relacionados con los hábitos de la familia, actividades sociales, la estructura física del hogar y de la actividad laboral ${ }^{15}$. Al mismo tiempo tienen tendencia a poner las necesidades del enfermo por delante de las suyas, minimizando la severidad de sus problemas y olvidándose 0 tardando en resolverlos ${ }^{16}$, dando a lugar de este modo el "síndrome del cuidador" conocido en el ámbito de la enfermería como Cansancio en el Rol del Cuidador ${ }^{17}$. Esto depende tanto del apoyo social que percibe física y mentalmente, como de la edad y el estado de salud física y mental del paciente ${ }^{18}$. Por tanto, el hecho de que el paciente sea dependiente afecta directamente al familiar.

Otro factor importante, que puede tener repercusión en la sobrecarga del cuidador es la percepción que el propio paciente tiene de su calidad de vida. Hay que tener en cuenta, que el padecimiento de una enfermedad crónica y su inclusión en una terapia sustitutiva como es la DP va acarrear cambios en sus hábitos y estilo de vida; pudiendo ocasionarle trastornos emocionales y repercutiendo en CVRS ${ }^{19}$. Este término, es definido por la OMS como "la percepción del individuo de su posición en la vida en el contexto de la cultura y sistema de valores en los que vive y en relación con sus objetivos, expectativas, estándares y preocupaciones" y se relaciona con la autonomía e independencia del paciente ${ }^{20}$. Por consiguiente, la dependencia que tiene el paciente en TRS en diálisis puede afectar a su dependencia emocional y psicológica; y podría estar relacionada con la sobrecarga de su cuidador principal.

Por tanto, teniendo en cuenta además el hecho diferencial de que la DP ambulatoria necesita la colaboración de un familiar cercano o cuidador principal que se responsabiliza en la mayoría de los casos del tratamiento del paciente, y al carácter crónico de esta patología, el cuidador principal es una persona expuesta a la sobrecarga. Por todo ello, hemos elegido este tema teniendo en cuenta el papel tan relevante que tiene el cuidador del paciente en DP.

\section{Objetivos}

\section{Generales:}

- Identificar el grado de sobrecarga de los cuidadores de pacientes con DP en la Unidad de Gestión Clínica (UGC) de Nefrología del Hospital Universitario Reina Sofía (HURS) de Córdoba y su relación con la CVRS y la dependencia del paciente. 


\section{Específicos: \\ - Determinar en qué medida se produce la sobre- carga del cuidador. \\ - Comparar el nivel de sobrecarga en cuidadores entre el cuestionario de Zarit y el de cuestio- nario de sobrecarga de cuidadores de pacientes en DP.}

\section{Metodología}

\section{Ámbito del estudio}

El presente estudio se llevó a cabo en la UGC de Nefrología del HURS de Córdoba, previa autorización del director de esta UGC (Anexo 1) y con la colaboración del facultativo y enfermeras/os de la Unidad de DP.

\section{Población y muestra}

Se estudió a los 18 pacientes en tratamiento con DP y sus respectivos cuidadores, previa información del objetivo del estudio (Anexo 2) y la firma del consentimiento informado para participar en el mismo (Anexo 3). La cumplimentación de los distintos cuestionarios se realizó en las revisiones periódicas del paciente en la Unidad de DP en el Servicio de Nefrología del HURS de Córdoba. La muestra estudiada fue el total de la población de pacientes incluidos en programa: 12 hombres $(67 \%)$ y 6 mujeres (33\%) y sus respectivos cuidadores, 6 hombres (33\%) y 12 mujeres cuidadores $(67 \%)$. Se perdió un paciente por traslado a otro centro sanitario.

\section{Criterios de inclusión}

Paciente de DP con su cuidador principal que aceptaron participar en la investigación tras leer el consentimiento informado.

\section{Diseño}

Se realizó un estudio observacional descriptivo y transversal.

\footnotetext{
Variables del estudio

Las variables demográficas añadidas en el estudio fueron: edad y género del paciente; tiempo que dedica el cuidador al paciente, género, parentesco y si es el cuidador principal o tiene más cuidadores. Estas variables se obtuvieron en la entrevista personal, sin tener que acceder a la historia clínica del paciente. Las variables de resultado fueron: CVRS y dependencia en el paciente, y nivel de sobrecarga del cuidador principal.
}

\section{Instrumentos de medida}

Para determinar la CVRS del paciente se usó el cuestionario SF-36 (Anexo 4) validado al español por Alonso y cols (21). Este cuestionario consta de 36 ítems, los cuales, se encuentran agrupadas en 8 dimensiones: función física, rol físico, dolor corporal, salud general, vitalidad, función social, rol emocional y salud mental. Tiene un rango de valor de 0-100. A menor puntuación, menos CVRS; y a mayor puntuación, mayor CVRS (22).

Para la medida del nivel de sobrecarga del cuidador, se utilizó el cuestionario de Zarit (Anexo 5), que es un cuestionario genérico adaptado al español por Martín y otros colaboradores (23). Consta de 22 ítems con 4 opciones de respuesta desde $0=$ nunca hasta $4=$ casi siempre. Los puntos de corte para determinar el grado de sobrecarga sería: no sobrecarga $=46-47$, sobrecarga leve $=46-47$ y sobrecarga intensa $=>56$ (24). Es importante destacar que estos dos cuestionarios son instrumentos de valoración para enfermería que se utilizan en el Servicio Andaluz de Salud y éste último está a disposición de todos los profesionales en la Web de este organismo (25).

Además, se utilizó un cuestionario específico para analizar la sobrecarga de familiares de pacientes en DP, y que al mismo tiempo evalúa la dependencia del paciente según la percepción del cuidador (Anexo 6). Este cuestionario ha sido utilizado en nuestro país, por Teixidó y cols (26) y contiene 30 ítems agrupadas en 3 escalas que se denominan: Escala de dependencia, Escala de sobrecarga del cuidador, Escala de repercusiones socio-familiares sobre el cuidador. En la segunda escala se refiere a la sobrecarga subjetiva del cuidador mientras que la tercera escala se refiere a la objetiva. Para obtener la sobrecarga total se suman ambas escalas con un rango de valor de 22110. A menor puntuación, menos sobrecarga; y a mayor puntuación, mayor sobrecarga.

\section{Recogida de los datos}

Los datos se obtuvieron en la semana, en la que los pacientes con sus cuidadores asistieron a la revisión mensual. En esta revisión, los pacientes y sus cuidadores están una media de 3-4 horas en la Unidad de DP, por lo cual no ha sido necesario añadir tiempo extra para la cumplimentación de los cuestionarios.

\section{Análisis estadístico}

Para las variables cualitativas se utilizó la distribución de frecuencias, mientras que para las variables numéricas se usó la media \pm la desviación 
estándar de la media. Para la relación entre variables se utilizó el test de Correlación de Pearson o el Test Spearman según los datos siguieron o no una distribución normal. Los datos se analizaron con el paquete estadístico SPSS 18.0.

\section{Consideraciones éticas}

Para llevar a cabo esta investigación, se solicitó la aprobación del Comité de Ética e Investigación Sanitaria del HURS de Córdoba. Para ello, entregamos la documentación solicitada en el Portal de Ética de la Investigación Biomédica de Andalucía.

Una vez obtenido su aprobación el 30/04/15 (Anexo 7), se comenzó a proporcionar los cuestionarios a los cuidadores y pacientes. Se garantizó en todo momento el anonimato tanto del paciente y cuidador principal como la confidencialidad de la información recogida, según la Ley Orgánica de Datos de Carácter Personal 15/99.

\section{Resultados}

La edad media de la población estudiada fue de $66,5 \pm 16,17$, de los cuales, el $33 \%$ eran hombres y el $67 \%$ eran mujeres.

Respecto a la CVRS, en la mayoría de las dimensiones (tabla 1), se observó que está disminuida pero no de manera importante. Las de menor puntuación fueron la de salud en general con un $63,89 \%$ y la de Vitalidad con un $66,67 \%$.

A continuación, dichas dimensiones se agruparon en dos componentes fundamentales. En el componente de salud física se seleccionó:
Tabla 1. Valor medio de las dimensiones de SF-36

\begin{tabular}{|l|c|c|c|c|}
\hline & Función Física & Salud General & Rol físico & Dolor corporal \\
\hline Media & 58,89 & 63,89 & 75,69 & 84,72 \\
\hline $\begin{array}{l}\text { Desviación } \\
\text { estándar }\end{array}$ & 27,362 & 19,596 & 29,149 & 24,463 \\
\hline & Vitalidad & Función Social & Rol emocional & Salud Mental \\
\hline Media & 66,67 & 91,67 & 90,73 & 73,61 \\
\hline $\begin{array}{l}\text { Desviación } \\
\text { estándar }\end{array}$ & 15,309 & 24,254 & 24,573 & 20,920 \\
\hline
\end{tabular}

función física, rol físico, dolor corporal y salud general. Mientras que en el componente de salud mental se introdujo: vitalidad, función social, rol emocional y salud mental. La media total de ambas dimensiones se observa en la tabla 2. Con estos resultados, se verificó una calidad de vida disminuida en el paciente tanto en su condición física con un $70,79 \pm 22,37$ como en su condición mental con un $80.66 \pm 19,77$.

Tabla 2. Valores del SF-36 con la puntuación obtenida de los pacientes en ambos componentes

\begin{tabular}{|l|c|c|}
\hline & $\begin{array}{c}\text { Componente } \\
\text { Físico General }\end{array}$ & $\begin{array}{c}\text { Componente } \\
\text { Social General }\end{array}$ \\
\hline Media & 70,7986 & 80,6694 \\
\hline $\begin{array}{l}\text { Desviación } \\
\text { Estándar }\end{array}$ & 22,37769 & 19,77297 \\
\hline
\end{tabular}

Cuando se compararon los valores en todos los componentes del SF-36, con los valores ajustados para la población general, se puede observar que la mayoría de los resultados son similares excepto en la función física y el componente de salud física y mental general (figura 1).

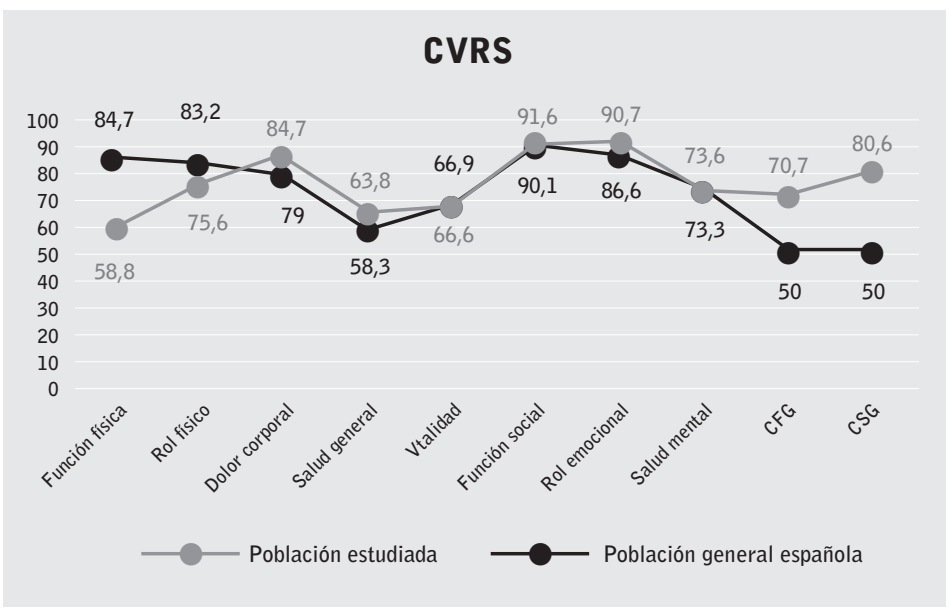

Figura 1. Valor medio de las dimensiones SF-36 de pacientes en DP y población española general
Por otro lado, podemos observar en la figura 2, los valores de la variable dependencia, en la que observamos que hay un $16,67 \%$ de pacientes dependientes en DP y el $83,33 \%$ restante, que son independientes.

Con respecto a los cuidadores, un 33\% eran hombres y un $67 \%$ eran mujeres, siendo el tiempo medio de cuidado que dedicaban al paciente de 14,78 $\pm 9,68$ horas al día.

Con respecto al cuestionario de sobrecarga de Zarit, se puede observar el grado de sobrecarga 
del cuidador en las tres categorías correspondientes representadas en la figura 3 . Se determinó una sobrecarga intensa del $11,1 \%$, una sobrecarga moderada del $16,7 \%$ y un grado de no sobrecarga del $72,22 \%$.

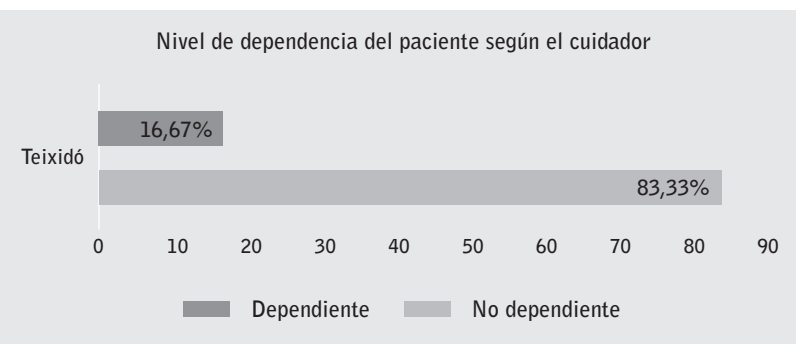

Figura 2. Dependencia del paciente según el criterio del cuidador.

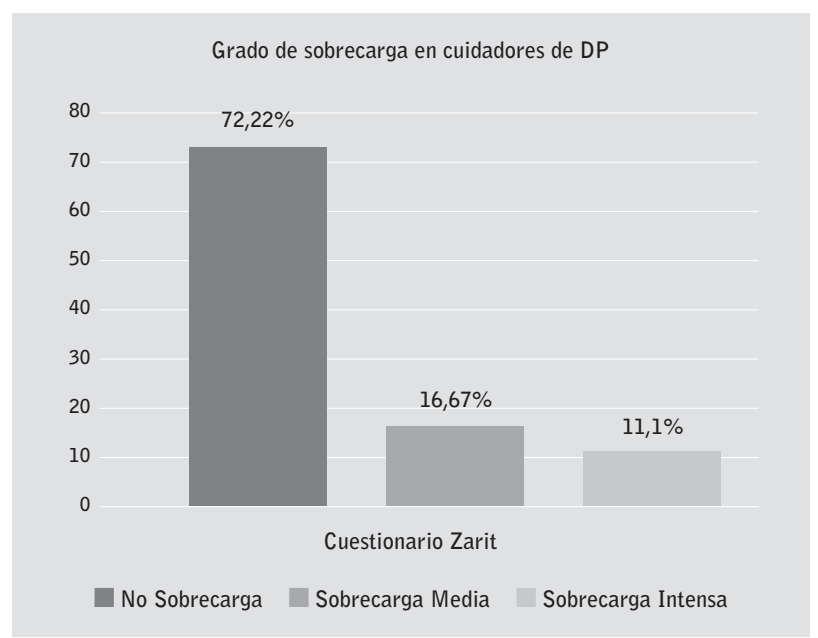

Figura 3. Distribución de los cuidadores según el cuestionario de Zarit.

Ante el escaso tamaño de la muestra se decidió agrupar los resultados del Zarit en dos categorías, para determinar el porcentaje de cuidadores con algo de sobrecarga y los que no tenían esta percepción, tal como puede observarse en la figura 4.

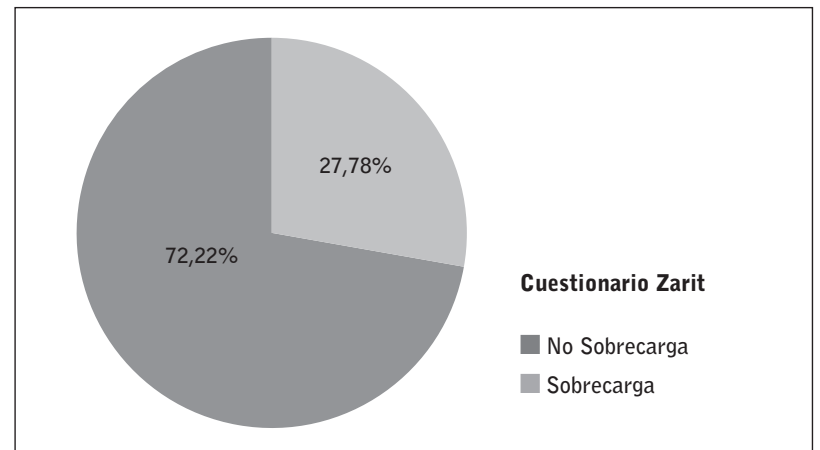

Figura 4. Sobrecarga de los cuidadores según el cuestionario de Zarit.
Cuando se analiza la variable sobrecarga con el Cuestionario de Sobrecarga específico para los cuidadores de pacientes en DP, tal como se observa en la figura 5, se encontró un grado de sobrecarga muy similar respecto al de Zarit. Hubo una pequeña diferencia en la clasificación no sobrecarga de un $66,67 \%$ a un $72,22 \%$ respecto al $33,33 \%$ y $27,78 \%$ de sobrecarga, lo que correspondería una diferencia en un cuidador.

Cuando se correlacionó los resultados de ambos cuestionarios para medir sobrecarga, se encontró una correlación estadísticamente significativa entre ambos (coeficiente de correlación de Spearman de 0,875, $\mathrm{p}=0.000$ ). Es decir, hubo una muy buena correlación entre ambos cuestionarios, lo cual es digno de destacar, pues uno es genérico y otro es un cuestionario específico para pacientes en DP.

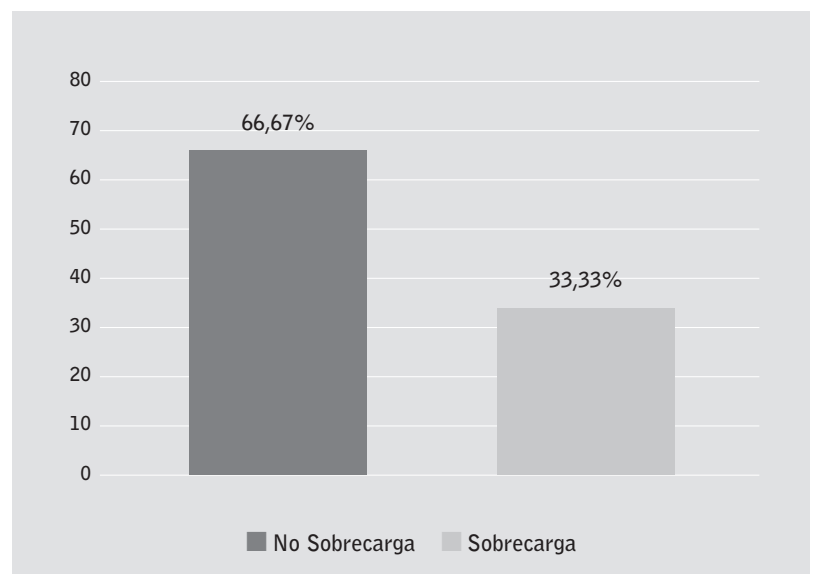

Figura 5. Distribución de los cuidadores según el cuestionario de sobrecarga específico para los cuidadores en DP.

No se encontraron ninguna otra relación entre las variables.

\section{Discusión}

El objetivo del presente estudio era analizar la relación del grado de sobrecarga del cuidador principal con la dependencia y la CVRS del paciente en tratamiento con DP, debido a la hipótesis que se planteó sobre una posible relación directa entre las variables nombradas.

Para evaluar el síndrome del cuidador se deben explorar diferentes campos, entre los que destacan: los aspectos de sobrecarga, el abandono del autocuidado, la vergüenza ante el comportamiento del enfermo, la irritabilidad ante la presencia del enfermo, el miedo por 
los cuidados o el futuro familiar, la pérdida de rol social y familiar ante los cuidados del familiar y el sentimiento de culpabilidad por no hacer lo suficiente. La escala capaz de evaluar todo esto es el cuestionario Zarit ${ }^{27}$. Sin embargo, otro cuestionario que hemos en encontrado en nuestra búsqueda bibliográfica es el cuestionario específico para cuidadores en DP utilizado en España por Teixidó. Éste, además de evaluar los campos anteriormente nombrados, también mide los específicos en DP como por ejemplo el sentimiento de culpa del cuidador ante una infección del catéter del paciente ${ }^{26}$.

En nuestra muestra, hemos utilizado ambos cuestionarios para, comprobar la utilidad del Zarit en este tipo de cuidadores, y hemos podido comprobar que tienen una muy buena correlación $(r=0,875)$; es decir, ambos son igual de válidos para determinar el grado de carga del cuidador principal del paciente en DP. Este dato nos parece interesante, ya que aunque el Zarit es un cuestionario genérico, es útil también para medir al nivel de sobrecarga en los cuidadores de los pacientes en DP, teniendo en cuenta que esta modalidad de terapia renal sustitutiva es muy específica y el rol del familiar es fundamental. No obstante, no hemos encontrado muestras investigadas con ambos cuestionarios para poder contrastar estos resultados.

Por otro lado, nuestros resultados muestran una puntuación media de sobrecarga de 39,22 $\pm 11,471$ con Zarit y $41,89 \pm 14,93$ con el cuestionario específico para pacientes en DP, cuya clasificación se corresponde con no sobrecarga. Éstos resultados de sobrecarga en DP son similares a los encontrados por otros autores, en los que se han usado indistintamente un cuestionario u otro. En el estudio de Teixidó se identifica un bajo número de cuidadores con sobrecarga respecto al total de la muestra ${ }^{26}$. En otro estudio, en una muestra estudiada con el cuestionario de Zarit se también se encontró distinguió un porcentaje importante de cuidadores con no sobrecarga respecto a los que si la presentaban ${ }^{28}$.

En relación con el nivel dependencia del paciente, se observó una puntuación media de $20,22 \pm 5,93$, cuya clasificación se corresponde con no dependiente, no encontrándose relación entre esta y el grado de sobrecarga del cuidador principal. Esto puede deberse al pequeño tamaño muestral, pues en otros estudios han comunicado esta relación ${ }^{29}$. Esto es lógico, debido a que al aumentar la edad de los pacientes, aumenta su dependencia y precisan en muchos casos de un cuidador que les preste apoyo o realice la técnica, originando que éstos estén expuestos a la sobrecarga.
Así lo corroboran los resultados de otro estudio, donde indica que hay mayor sobrecarga y peor CVRS del cuidador principal en diálisis cuanto menor apoyo social reciba y mayor sea la edad del paciente ${ }^{30}$. Según el estudio de Teixidó, las tareas de la DP no tienen relación con la sobrecarga del cuidador, sino que ésta deriva de la dependencia del paciente y de cómo viven ambos la responsabilidad del TRS ${ }^{26}$. Parece lógico por tanto, que los pacientes que tienen más dependencia sean los que presentan mayor sobrecarga en sus cuidadores, aunque esto no se haya confirmado en nuestra muestra.

Con respecto a la CVRS, evaluada con el cuestionario SF-36, se obtuvo una media de $70,79 \pm 22,37$ en el componente físico general y $80,66 \pm 19,77$ en el componente social general, cuya caracterización se corresponde con una CVRS por encima de la población general. Esto parece indicar que el paciente cuando empieza en DP, a pesar de tener los resultados de algunas dimensiones por debajo de la población en general, siente una gran mejoría en su vida de tal forma que ambos componentes se encuentran por encima de la población sana.

Cuando se relacionó la dependencia con la CVRS del paciente, no se encontró ninguna relación estadísticamente significativa, posiblemente también a la pequeña muestra que se ha podido incluir en el estudio. Por el contrario en otros estudios se ha documentado que la edad del paciente se relaciona con un aumento de la carga del cuidador y una disminución de la CVRS entre los pacientes y los cuidadores ${ }^{31}$. Sin embargo, es cierto que esta CVRS suele ser inferior a la de la población en general por la edad del paciente y no por el hecho de estar en DP32. Por lo que es lógico pensar que a mayor edad, más dependencia y por tanto menor CVRS, sin ningún tipo de relación con la DP.

No se encontró ninguna otra relación entre las variables estudiadas, posiblemente a causa de que la muestra es pequeña. Ésta junto a la poca información que hay del presente estudio y no saber con exactitud los puntos de corte de los niveles de sobrecarga del cuestionario específico en DP, han sido las limitaciones más importantes que se han encontrado durante el desarrollo de este trabajo. No obstante, ha sido imposible aumentar la muestra pues se ha estudiado a toda la población que en el momento de recibir la autorización del Comité de Ética e Investigación estaba en programa de DP en la UGC de Nefrología del HURS de Córdoba; pues la gran mayoría de los enfermos con ERC están en programa de HD periódicas en este medio. Por consiguiente, sería interesante realizar un estudio multicéntrico entre pa- 
cientes de DP de diferentes hospitales y observar si la relación de entre nuestras variables coincide.

A la vista de estos resultados, podemos concluir que, al menos en la muestra estudiada se encuentra una baja proporción de cuidadores con sobrecarga. De igual forma, los pacientes presentan un nivel bajo de dependencia con una CVRS similar a la población general de referencia excepto en la función física.

Recibido: 25 julio 2015

Revisado: 2 agosto 2015

Modificado: 5 agosto 2015

Aceptado: 6 agosto 2015

\section{Bibliografía}

1. Gómez FG, Juán LC, Vizoso RP, Fraga IF, Barcia $M M$, Rico LP, et al. Las cuidadoras de enfermos en diálisis peritoneal, invisibilidad de un problema. [citado 15 de febrero de 2015]; Recuperado a partir de: http://www.revistaseden.org/files/ las\%20cuidadoras.pdf

2. Soriano S. Definición y clasificación de los estadios de la enfermedad renal crónica. Prevalencia. Claves para el diagnóstico precoz. Factores de riesgo de enfermedad renal crónica. Nefrología, 2004; 24 Supl. 6: 27-35.

3. Registro SEN. Sociedad Española de Nefrología [Internet] [citado 30-04-2015] Recuperado a partir de: http://www.senefro.org/modules/ webstructure/files/inforreercongsen2014final.pdf

4. ERA-EDTA Registry 2012, AnnualReport. [Internet] [citado 01-05-2015] Recuperado a partir de: http://www.era-edta-reg.org/files/ annualreports/pdf/AnnRep2012.pdf

5. Periz LA, Armangué PG, Sánchez-Salido LT. Visión enfermera de las necesidades psicosociales de los cuidadores de personas en tratamiento con Diálisis Peritoneal. Revista de la Sociedad Española de Enfermería Nefrológica. 2007;10(1):47-52.

6. Martín Espejo JL, Gómez Castilla AC. Enseñanza de diálisis peritoneal continua ambulatoria. En:

Coronel F, Montenegro J, et al, editores. Manual Práctico de Diálisis Peritoneal. $2^{\mathrm{a}}$ ed. Barcelona: Atrium Comunicación Estratégica; 2005. p. 299306.

7. Vega Díaz N, Gallego Samper R, Palop Cubillo L, Henríquez Palop F. Diálisis Peritoneal Automatizada. En: Coronel F, Montenegro J, et al, editores. Manual Práctico de Diálisis Peritoneal. $2^{a}$ ed. Barcelona: Atrium Comunicación Estratégica; 2005. p. 59-68.

8. Ramírez VJA, del Río BR, Russell MER, López CGF. Validez de la Entrevista de Cargade Zarit en una muestrade cuidadores primarios. Psicología y Salud, julio-diciembre, año/vol. 18, número 002 Universidad Veracruzana Xalapa, México. Psicología y Salud. 2008;18(2):237-45.

9. Linde JMM, Velasco M de los ÁI, Velasco BI. El apoyo social como modulador de la carga del cuidador de enfermos de Alzheimer. Psicología y salud. 2014;15(1):33-43.

10. Montero Pardo $X$, Jurado Cárdenas $S$, Valencia Cruz A, Méndez Venega J, Mora Magaña I. Escala de carga del cuidador de Zarit: evidencia de validez en México. Psicooncología [Internet]. 10 de junio de 2014 [citado 4 de marzo de 2015];11(1). Recuperado a partir de: http://revistas.ucm.es/ index.php/PSIC/article/view/44918.

11. Jofré Aravena V, Sanhueza Alvarado 0. Evaluación de la sobrecarga de cuidadoras/es informales. Ciencia y enfermería. 2010;16(3):111-20.

12. Suri RS, Larive B, Garg $A X$, Hall $Y N$, Pierratos $A$, Chertow GM, et al. Burden on caregivers as perceived by hemodialysis patients in the Frequent Hemodialysis Network (FHN) trials. Nephrology Dialysis Transplantation. 1 de julio de 2011;26(7):2316-22.

13. Contreras Gallego A, López Soto P, Crespo Montero R. Análisis de la relación entre nivel de dependencia del paciente en hemodiálisis y sobrecarga del cuidador principal. Enfermería Nefrológica. 2014;17(2):98-103.

14. Gil Barrera I, Pilares Puertas JA, Romero López $\mathrm{R}$, Santos Seco M. Cansancio del rol de cuidador principal del paciente en hemodiálisis. Enfermería Nefrológica. 2013;16:172-3. 
15. Cesar ED, Beuter M, Brondani CM, Pauletto MR, Timm AMB, Jacobi $C$ da S. A diálise peritoneal na vivência de familiares cuidadores. Revista da Rede de Enfermagem do Nordeste-Rev Rene [Internet]. 2013 [citado 25 de febrero de 2015]; 14(3). Recuperado a partir de: http://www.revistarene. ufc.br/revista/index.php/revista/article/view/1063.

16. Rabow MW, Hauser JM, Adams J. Supporting family caregivers at the end of life: They don't know what they don't know. Jama. 2004;291(4):483-91.

17. Flores E, Rivas E, Seguel F. Nivel de sobrecarga en el desempeño del rol del cuidador familiar de adulto mayor con dependencia severa. Ciencia y enfermería. 2012;18(1):29-41.

18. Alvarez-Ude F, Valdés $C$, Estébanez $C$, Rebollo $P$, Group FS, others. Health-related quality of life of family caregivers of dialysis patients. J Nephrol. 2004;17(6):841-50.

19. Contreras F. Quality of Life and Adherence in Patients with Chronic Kidney Disease in Haemodialysis Treatment. Universitas Psychologica. 2006;5(3):487-99.

20. Rodríguez E, Aja Crespo A, Saenz de Buruaga Perea $A$, Incera Setién $M$, et al. Factores sociológicos y calidad de vida relacionada con la salud en pacientes en hemodiálisis. Revista de la Sociedad Española de Enfermería Nefrológica. 2011;14(2):98-104.

21. Rodríguez Vidal $M$, Castro Salas $M$, Merino Escobar JM. Calidad de vida en pacientes renales hemodializados. Ciencia y enfermería. $2005 ; 11(2): 47-55$.

22. Vilagut $G$, Ferrer $M$, Rajmil $L$, Rebollo $P$, Permanyer-Miralda G, Quintana JM, et al. El cuestionario de salud SF-36 español: una década de experiencia y nuevos desarrollos. Gaceta sanitaria. 2005;19(2):135-50.

23. Painepán B, Kühne W. Sobrecarga, afrontamiento y salud en cuidadoras de pacientes con demencia tipo Alzheimer. Summa psicológica UST (En línea). 2012;9(1):5-14.

24. De Santander C. El cuestionario de sobrecarga del cuidador de Zarit. Cómo administrarlo e interpretarlo. 2008 [citado 25 de mayo de 2015]; Recuperado a partir de: http://scielo.isciii.es/pdf/ gs/v22n6/cartal.pdf.
25. Cuestionarios, test e índices de valoración enfermera en formato para uso clínico - Servicio Andaluz de Salud [Internet] [citado 2 de mayo de 2015]; Recuperado a partir de: http://www. juntadeandalucia.es/servicioandaluzdesalud/ principal/documentosAcc.asp? pagina=pr_desa_ Innovacion5.

26. Teixidó J, Tarrats $L$, Arias $N$, Cosculluela $A$. Overload questionnaire for caregivers of patients on peritoneal dialysis. Nefrología [Internet]. 2006 [citado 5 de febrero de 2015];26(1). Recuperado a partir de: https://revistanefrologia. com/revistas/P-E/P-E-S-Al174-EN.pdf.

27. Vélez Lopera JM, Berbesí Fernández $D$, Cardona Arango D, Segura Cardona A, Ordóñez Molina J. Validación de escalas abreviadas de zarit para la medición de síndrome del cuidador primario del adulto mayor en Medellín. Atención Primaria. julio de 2012;44(7):411-6.

28. Castro CC, Quiroz LMH, Mosquera DP, Ruiz AS. Calidad de vida relacionada con la salud de los pacientes y sus cuidadores en una unidad renal de la ciudad de Cali en 2013. Ciencia \& Salud. 2014;2(8):29-35.

29. López G, del Valle M, Sesmero Ramos $C$, Portolés Pérez JM, de Enfermería DU, de Nefrología S. Estudio sobre el cambio de elección de tratamiento renal sustitutivo en pacientes que han optado por diálisis peritoneal. Revista de Enfermería Nefrológica [Internet]. 2006 [citado 15 de mayo de 2015];9. Recuperado a partir de: http://scielo.isciii.es/pdf/nefro/v9nl/art01.pdf.

30. Alvarez-Ude F, Valdés C, Estébanez C, Rebollo P, Group FS, others. Health-related quality of life of family caregivers of dialysis patients. J Nephrol. 2004;17(6):841-50.

31. Shimoyama S, Hirakawa 0, Yahiro K, Mizumachi T, Schreiner A, Kakuma T. Health-related quality of life and caregivers burden among peritoneal dialysis patients and their family caregivers in Japan. Peritoneal Dialysis International. 2003;23(Supplement 2):S200-5.

32. Segura M, Espejo J. Salud percibida por los pacientes en DPCA y DPA. Revista Sociedad española de enfermería y nefrología. 2008;11(2):102-9. 